\title{
The annual meeting of the British Society of Gastroenterology
}

The annual meeting of the British Society of Gastroenterology was held at King's College Hospital, London, on 4 and 5 November 1960 under the Presidency of Mr. Harold Edwards, with Dr. Bruce Pearson as the local secretary.

At the general business meeting the following elections were made: President, E. Bulmer; Treasurer, Hermon Taylor; Secretary, W. A. Bourne; Council Members, S. Sherlock and S. C. Truelove. The following were elected full members of the Society: Michael Atkinson, D. H. Cummack, David Douglas, C. W. A. Falconer, A. P. M. Forrest, Terence Fulton, J. B. Gibson, R. H. Girdwood, R. A. MacMynn, J. G. Murray, W. A. B. Nicholson, Brian Schofield, Geoffrey Slaney, and Ian Todd. The following were elected as associate members: E. D. Acheson, H. T. F. Barniville, E. V. Cox, C. W. Crane, G. P. Crean, I. E. Gillespie, F. E. Higgins, I. D. A. Johnston, W. G. Manderson, G. F. Matts, A. G. Melrose, Annette Rawson, H. I. Tankel, and R. S. Williams.

It was decided that the Society should play a more active role in international relations in respect of gastroenterological work and that there was a need to keep in touch with other international societies and to assist in the coordination of education and research activities. It was decided to form a special committee with T. C. Hunt, F. Avery Jones, Hermon Taylor, and Geoffrey Watkinson as members.

On the Friday evening a most excellent dinner was held at the Apothecaries Hall and this had been arranged by Professor Magnus. The President spoke of the work of the Society and welcomed the two visitors, Dr. Grossman and Sir William Morrow.

After dinner the Clerk of the Apothecaries gave an account of the history of the beautiful hall.

\section{Scientific meeting}

$$
\begin{gathered}
\text { THE EFFECT OF PROSTIGMINE ON } \\
\text { HUMAN COLONIC MOTILITY }
\end{gathered}
$$

N. A. CHAUDHARY AND S. C. TRUElove (OXFORD) had made a comparative study of the patterns of colonic motility following a standard intramuscular dose of prostigmine methylsulphate in normal subjects, patients with irritable colon syndrome, and patients with ulcerative colitis. In normal subjects, the standard dose of prostigmine methylsulphate provoked increased activity with a resulting pattern not dissimilar from that of the resting pattern of patients with spastic colon in a stage of symptoms. Patients in all clinical categories of the irritable colon syndrome displayed more colonic activity after prostigmine than normal subjects. This was so whether or not they were in a stage of symptoms. By contrast, the response of patients with ulcerative colitis to a standard dose of prostigmine was little if any different from that of normal subjects. These results suggest that patients with spastic colon are subject to excessive parasympathetic stimulation or to increased reactivity to normal stimuli.

RADIOLOGICAL AND MANOMETRIC STUDIES OF CHANGES IN MOTILITY OF THE ALIMENTARY TRACT IN DIFFUSE SYSTEMIC SCLEROSIS AND POLYMYOSITIS

D. A. W. EDWARDS (LONDON) said that in a group of 21 patients with scleroderma, dermatomyositis, polymyositis, or systemic sclerosis, the motor activity of the gut had been examined by radiology and by recording changes in intraluminal pressure with open tubes and miniature balloons. The majority of patients were investigated personally; information about the others was obtained from hospital records. In all but two patients there was evidence of a marked decrease in smooth muscle activity in some part of the gut, and, in a small group in which histological material became available, a decrease in the amount of smooth muscle was demonstrated.

The commonest disturbance was loss of oesophageal peristalsis, sometimes associated with stiffening of the lower end of the oesophagus and loss of tone in the cardiac sphincter. Changes in motility of the stomach were difficult to distinguish from the normal, but the characteristic squashed out atonic immotile duodenal loop was present in six out of 13 patients adequately examined. A similar appearance of flattened immotile loops of small intestine was seen in five out of 10 patients. Five out of six patients had saccular dilatations and a disorganized haustral pattern in the colon.

The characteristic changes are valuable as a diagnostic aid when biochemical or external manifestations of this disease complex are absent or equivocal, or when the disease presents as a gastroenterological problem. Loss of smooth muscle appears to be an integral part of the disease process.

\section{GASTRIC SECRETION IN LIVER DISEASE}

SOAD TABAQCHALI AND A. M. DAWSON (LONDON) It has been claimed that there is a high incidence of peptic 
ulcer in patients with portal cirrhosis. Recently, it has been suggested that this might be related to the fact that in dogs which have undergone a portal systemic shunt procedure there is a consistent increase in acid secretion from a Heidenhain pouch.

The records of 200 consecutive patients with portal cirrhosis were analysed and a probable increased incidence of peptic ulcer, especially in women, was found, in contrast to the greatly increased incidence in patients with primary biliary cirrhosis. Gastric secretory function was assessed in patients with portal cirrhosis, some of whom had had a portacaval anastomosis operation, and in patients with primary biliary cirrhosis. This was done by measuring basal acid secretion, maximal histamine secretion, and the 24-hourly gastric $p \mathrm{H}$. In all groups the maximal response to histamine was normal. However, in patients with a portacaval anastomosis there was a tendency to a low gastric $p \mathrm{H}$ at night, whereas patients with uncomplicated portal cirrhosis and primary biliary cirrhosis did not differ from the controls in this respect.

\section{A POSSIBLE EXPLANATION OF FAILURE OF RADIO- TRIOLEIN TO DIAGNOSE STEATORRHOEA}

A G. COX (SHEFFIELD) In a clinical trial, radio-iodinated triolein failed to diagnose steatorrhoea in $50 \%$ of cases with a high chemical faecal fat excretion. A possible explanation was instability of the radio-iodine label in the alimentary tract. To investigate this point, a method using an anion exchange resin was evolved and applied in vitro. When incubated with duodenal juice, six batches of triolein tagged with the standard $\mathrm{I}^{131} \mathrm{Cl}$. label showed a steady rise in the amount of the radio-isotope liberated. This demonstration of the instability of the label requires study in vivo. The method also confirmed that the simple $I^{131}$ label, still occasionally used, is highly unstable.

In the discussion $\mathrm{N}$. Coghill noted that Lubran, working with him, had obtained good correlation and it was agreed that results varied appreciably in different places. Cooke pointed out that strictly speaking steatorrhoea was demonstrated and not diagnosed.
OBSERVATIONS ON THE CLINICAL TESTING OF GASTRIC ANTISECRETORY DRUGS AND ANTACIDS

J. E. LENNARD-JONES (LONDON) had studied the effect of two gastric antisecretory drugs, a long-acting form of propantheline bromide and poldine methosulphate (Nacton), on the $p \mathrm{H}$ of hourly samples of gastric contents in patients with uncomplicated duodenal ulcer. Both the drugs significantly reduced the acidity of the sample when patients took hourly drinks of milk-cream without other food. When the same patients took a gastric-type diet, a small reduction in acidity was demonstrable with propantheline but no reduction with Nacton. In other patients taking a diet, Nacton given alone did not reduce acidity even when given in doses large enough to cause unpleasant side-effects. Nacton potentiated the effect of suboptimal doses of alkali and the combination reduced acidity during waking hours. Although Nacton delayed gastric emptying at night, the effect of a dose of alkali at bed-time was not prolonged. When patients sucked antacid tablets continuously a satisfactory reduction in acidity of the gastric contents during waking hours was achieved without the use of an antisecretory drug.

From this work it is concluded that the two antisecretory drugs studied are less effective in reducing the acidity of gastric contents under the conditions of clinical use than under experimental conditions. Antacids, sucked as tablets, satisfactorily reduce gastric acidity during the day; the two antisecretory drugs do not usefully reduce the $p \mathrm{H}$ of the gastric contents at night when a patient is taking a gastric-type diet.

In the discussion J. N. Hunt noted that the antisecretory drugs had been given for a relatively short time, about five days before the observations were made, and suggested that longer prior administration might have produced a different result. The speaker did not support this view.

Sircus commented on the complexity of the factors resulting in the final sampling figure.

\section{SYMPOSIUM ON APPLIED PHYSIOLOGY OF THE STOMACH}

There were four opening papers and these were given by Professor R. A. Gregory, J. G. Murray, M. R. Vane, and W. I. Card.

\section{THE PHYSIOLOGY OF GASTRIC SECRETION}

PROFESSOR R. A. GREGORY (LIVERPOOL) Studies during the last 10 years by Uvnas, Dragsted, Schofield, Gregory, and Tracy and others have led to a general acceptance of the suggestion first made by Straaten that vagal activity is important for the release of gastrin from the pyloric antrum. It is now agreed that in addition to its direct action on the gastric glands the vagus also excites the antrum and thus contributes, in combination with local stimulation by the swallowed food, to release the pyloric hormone. The 'cephalic phase', defined originally by Pavlov, thus involves a hormonal component. How long the release of gastrin continues is uncertain, but two important controlling factors are now recognized to exist. One is the intragastric accumulation of acid which inhibits further release of gastrin by a local action on the pyloric mucosa. The other, as recently shown by Gregory and Tracy, is the action of the hormone enterogastrone liberated from the duodenum by such agencies as fat, acidity, and hypertonicity of the duodenal contents.

In addition to this hormonal mechanism, reflex control of gastric secretion from the duodenum undoubtedly exists, and the vagi are probably the efferent pathway, as 
indicated by the studies of Code and Watkinson. The highly purified preparation of gastrin obtained by Gregory and Tracy stimulates acid but not pepsin secretion in the conscious human subject, as well as in animals. Its action resembles that of histamine, but differs from this substance in that it is strongly inhibited by atropine, and single intravenous injections are effective in exciting secretion.

\section{NERVOUS CONTROL OF GASTRIC FUNCTION}

J. G. MURRAY (LONDON) Recent experimental work has thrown new light on the composition of the vagus nerve. About 80 to $90 \%$ of the fibres supplying the abdominal viscera are afferent, some arising from stretch receptors and others from chemoreceptors in the stomach.

Combined anatomical and physiological observations are consistent with the view that the intrinsic nerve plexuses contain local reflex arcs and mediate, for example, peristalsis. At least two sets of enteric neurons are involved: (a) sensory neurons, the processes of which are distributed to the mucous membrane on the one hand, and to motor neurons on the other, and $(b)$ motor neurons which innervate the muscle coats. Of all the nervous tissue in the stomach and gut, by far the largest part is of intrinsic origin.

Lastly, the surprising plasticity of the autonomic nervous system following injury or disease has only been appreciated over the last few years. If an organ is partially denervated, then the remaining intact nerve fibres are stimulated to give off tiny branches. These sprouts connect up with denervated effector organs and result in return of function. Even after complete denervation, nearby nerve fibres may sprout to produce quite bizarre effects. This phenomenon was briefly described with particular reference to vagotomy.

\section{THE PHARMACOLOGY OF THE SMOOTH MUSCLE OF THE STOMACH}

J. R. VANE (LONDON) Dale and Feldberg in 1934 demonstrated that excitation of the vagus nerves brought about the release of acetylcholine in the wall of the stomach. However, the suggestion has often been made that vagal effects in the stomach are mediated by the release of histamine. This suggestion was based on the high potency of histamine as a stimulus for gastric secretion and for muscular contraction. Moreover, histamine is normally present in the stomach wall. More recently, another hormone, 5-hydroxytryptamine, had been isolated from the stomach. In consequence, the roles of acetylcholine, histamine, and 5-hydroxytryptamine have been re-investigated.

The release of acetylcholine from parasympathetic nerve endings has been confirmed. It has also been shown that the major effect of histamine on the smooth muscle of the stomach, which is to increase its tone, is brought about through an excitation of nerves.

\section{CLINICAL APPLICATION}

W. I. CARD (EDINBURGH) underlined the ideas found valuable in gastric physiology of the last 10 years. The most important general advance in the last 10 years has been the increasing application of the scientific method to gastroenterology, and much of this advance has been due to the introduction of better investigative techniques.

With the introduction of the electron microscope morphology has come into its own again, revealing almost a new dimension of thought. The obstacle here seems to be not that of resolution but of finding methods of differentiating structure.

Gastric secretion continues to command much of our attention, partly because it is simplest to measure and partly because high secretions play a part in the causation of certain peptic ulcers. The evidence that histamine is the actual pharmacological stimulant of the parietal cells continues to accumulate. The problem that has teased the physiologist for so long of inferring from the mixed gastric secretion the original constituents as secreted continues to appear insoluble. Over the last 20 years acid output rather than acid concentration in gastric juice has been generally adopted. Though the output is reproducible with the ordinary doses of histamine, the use of 'maximal' histamine stimulation does allow comparisons of one subject with another by excluding variations in one parameter, and also makes for a more rigorous definition of achlorhydria. The output of acid in a given time can be considered as the product of the rate of secretion and the number of secretory units, though the phrase 'rate of secretion' covers a number of factors. One of these which has been frequently overlooked is the blood flow to the mucosa which must, from consideration of energetics, be very large. Directly the acid output is shown to be related to the number of cells, the question immediately presents itself of accounting for the origin of a large cell mass. There seems to be a need to consider the existence and the role of trophic hormones, though it has not been possible to confirm the trophic action of histamine as had been previously reported. The possibility has to be considered that gastrin acts as a trophic hormone. The discovery of a gastrin-like hormone in the Zollinger-Ellison syndrome raised the question of whether this exists as a qualitatively distinct syndrome, or whether it is simply a grossly exaggerated form of a normal mechanism. Another hormone with a trophic action that had to be considered is cortisone. It has been shown that complete achlorhydria occurs in Addison's disease, that this may disappear after treatment with corticoids, and that even peptic ulceration may follow. Cortisone, given in an acute experiment, did not seem to produce an increased response to histamine in human subjects, but given over a longer period, as in the treatment of Crohn's disease, it undoubtedly increases the output of acid. This can only be due to an increase in the number of secretory units per cell, an increased number of cells, or an augmentation of secretory rate. The pituitary might also produce a trophic hormone from the gastric mucosa. There was atrophy in clinical hypopituitarism, a diminution of the parietal cell count had been demonstrated following experimental hypophysectomy, while the prolonged administration of corticotrophin in man produced increasing amounts of acid which it was difficult to believe could be due to anything else except hyperplasia of the parietal cells. 


\section{DISCUSSION}

Professor A. W. Kay commented that Mr. Murray's fascinating studies on the intimate neuroanatomy of the stomach had an obvious bearing on the operation of surgical vagotomy. His demonstration of 'sprouting' from the divided ends of the vagal fibres rather suggested an appreciable chance of vagal regeneration with a risk of late recurrent ulceration. In this connexion it was of interest that secretion studies had been recently performed in Sheffield on patients who had undergone vagotomy from a few months to some years previously. Analysis of these results showed that the great majority showed no tendency to a rising level of acid secretion and indeed there was a general trend towards a further reduction.

With regard to Professor Gregory's magnificent survey of the physiology of gastric secretion, we must have some concern about various discrepancies between the results of animal and human investigations. For example, antrectomy in the dog has been shown by both American and Scandinavian investigators to have no effect on histamine-provoked acid secretion. On the contrary, antrectomy in man causes a marked reduction not only of histamine secretion but of maximally stimulated acid secretion. It may be that we should give more attention to physiological studies in primates and there is certainly room for a great deal more investigation of patients undergoing planned surgery for duodenal ulcer.

The influence of the advances in physiology and pharmacology on surgical treatment demands that careful preoperative studies on gastric secretion should be carried out and thereby the operation selected most suited to the individual case. In gastrectomy, vagotomy with a drainage procedure, antrectomy with vagotomy, and gastrojejunostomy we have four operations of value in surgical therapy. Our task is to devise methods from which we can select the appropriate operation for each patient.

J. N. Hunt drew attention to the threshold for the effect of acid in reducing gastric secretion. Equal concentrations of hydrochloric acid, acetic acid, and citric acid were about equipotent, although the $p \mathrm{H}$ of the solutions were quite different.

Professor R. B. Welbourn referred to the influence of the major endocrine glands on gastric acid secretion, which Dr. Card mentioned. Hypophysectomy reduced secretion profoundly in the rat. Corticotrophin and growth hormone restored it. The latter observation does not appear to have been taken up by others. Pharmacological doses of cortisone, methyl prednisolone (a 'pure' glucocorticoid), and aldosterone all increase secretion similarly in the dog. The effect might be a mineral corticoid one, since methyl prednisolone has slight salt-retaining properties. However, they had recently investigated triamcinolone and dexamethasone, of which the mineralocorticoids were minimal, and had found that they also increased secretion. It seems likely, therefore, that the gastric activity of the adrenal steroids is not necessarily tied to their main metabolic actions. Cortisone increased by about $50 \%$ the maximal secretory capacity, in response to histamine, of Heidenhain pouches in dogs and it increased the parietal cell mass by a similar proportion. The physiological role of all these hormones appears to be 'permissive'.

M. I. Grossman said that the release of gastrin by vagal impulses had been unequivocally established. Schofield had placed the keystone in the arch of evidence supporting this conclusion. We should not, however, lose sight of the fact that vagal impulses stimulate the secretion of gastric acid by another mechanism, namely, direct cholinergic action on the acid-secreting glands. It is not yet possible to say which of these two vagal mechanisms for stimulation of acid secretion is quantitatively predominant.

Professor Gregory had introduced a new concept of the mode of action of enterogastrone. He believes that it inhibits gastric secretion by suppressing the release of gastrin. Inhibition of acid secretion by acid bathing the pyloric gland area is also attributable to prevention of gastrin release. Both of these mechanisms for suppression of gastrin release act at the final step in the process, that is, the release of gastrin by acetylcholine. It is unlikely that hydrogen ions penetrate to the site where cholinergic release of gastrin is occurring; therefore, it is postulated that acid releases a local humoral agent which interfers with gastrin release.

Professor L. J. Witts spoke of the value of the augmented histamine test in the differentiation of the anaemias. The essential defect in pernicious anaemia was loss of intrinsic factor but in adults it was excessively rare to find free $\mathrm{HCl}$ in a proved case of pernicious anaemia. In only four out of $\mathbf{3 0}$ patients with pernicious anaemia studied did the total volume of gastric juice after histamine exceed $20 \mathrm{ml}$. Electrometric measurements revealed a prehistamine juice more alkaline than $p \mathrm{H} 7.0$ in all but four instances and the lowest reading was $p \mathrm{H} \mathrm{6.71.}$ In only two patients did the $p \mathrm{H}$ change to the acid side after histamine and the shift was less than 0.33 units. In three there was no change in $p \mathrm{H}$. In all the others the $p \mathrm{H}$ rose after histamine and the mean maximum $p \mathrm{H}$ change indicated an increased alkalinity of 0.52 units. One of the most interesting studies at the moment was the detection of latent or preclinical forms of pernicious anaemia. As a rule 
the sequence was loss of $\mathrm{HCl}$, loss of intrinsic factor, and finally fall in serum vitamin $B_{12}$, but this order was not invariable.
Dr. Avery Jones commented on the value of the urinary pepsinogen test as a measure of gastric function.

\section{CHEST DISEASE AND PEPTIC ULCER}

B. G. RIDGEN (BRIGHTON) describes an investigation of 10,485 males of 21 years and upwards, attending a mass radiography unit, with the object of determining whether certain radiological abnormalities in the thorax were seen more commonly amongst those with a history of peptic ulceration than in those without such a history.

Each chest film was read in ignorance of the ulcer state; and each ulcer history was assessed in ignorance of the chest condition.

Of the 10,485 men, $409(3.9 \%)$ were deemed to have a valid history of peptic ulceration. The incidence of emphysema (without radiological evidence of focal lung disease) in the ulcer group was $6.35 \%$; in the remainder it was $1.87 \%$. Emphysema was thus seen more than three times as frequently in the ulcer patients. Intrathoracic tuberculosis (all stages) was seen in $10.75 \%$ of the ulcer group as against $6.55 \%$ of those without an ulcer history. Congenital abnormalities (of the ribs, heart, and lungs) were seen rather less commonly in the ulcer groups than in the others.

\section{METABOLIC INFLUENCES IN THE DISSEMINATION} OF CANCER

JOHN D. GRIFFITHS (LONDON) said that the effect of dietary deficiency in producing spontaneous tumours had been well described by Tannenbaum and Silverstone, who demonstrated that prolonged restricted caloric intake and malnutrition in laboratory animals would diminish induction and growth in spontaneous tumours. However, the influence of acute metabolic changes had not been investigated in relation to increased susceptibility of the host and its possible effect on the dissemination and subsequent implantation of neoplastic cells. In the present investigation the effect of 'acute' metabolic changes, such as starvation and dehydration, which can be considered a form of metabolic 'stress', had been studied in relation to the rats' susceptibility to inoculation of Walker 256 carcinosarcoma.

Results of the experiment indicated that starvation and dehydration for $\mathbf{4 8}$ hours did not increase susceptibility of the rat to subcutaneous inoculation of Walker tumour, but if starvation was continued for seven days there was an increase in percentage 'takes' of the tumour as compared with the incidence in normal healthy animals. There was also an increase in 'takes' of implanted tumour in young rats.

\section{CYTOLOGICAL STUDIES IN GASTRIC CARCINOMA}

M. K. MASON (LONDON) said the investigations were being made to assess the value of cytology in the early diagnosis of carcinoma of the stomach. Two methods of approach had been employed. The first consisted of gastric lavage using normal saline in suspected or known cases of cancer in order to identify any malignant cells exfoliated from the lesions. A number of difficulties had been encountered using this technique. The second method was to make smears from all stomach immediately after resection and to examine the slides for the presence of malignant cells. By this means a number of early surface carcinomas had been identified. These growths in general cannot be recognized macroscopically, and may be very small. By making smears, a considerably wider area of mucosa is covered than is possible with sections alone. Surface carcinomas appear to be commoner than is usually realized, and are easily overlooked without careful examination of the stomach.

\section{THE MEDIASTINUM IN PORTAL HYPERTENSION}

A. E. READ AND F. DOYLE (LONDON) had carried out a radiological study of the mediastinum in portal hypertension. The 'diameter' of the azygos vein had been measured, using tomography, in a series of patients with portal hypertension and in a group of normal controls. After correction for body weight differences a significant increase in the size of this vessel had been shown to exist in the portal hypertensive group. There was, however, no apparent correlation between the size of the azygos vein and various clinical manifestations of portal hypertension, such as the presence of ascites, encephalopathy, and gastrointestinal bleeding.

Measurement of the left paravertebral shadow had also shown that in portal hypertension this is sometimes widened. This measurement is, however, probably not a reliable indication of the size of the hemiazygos veins. A very wide left paravertebral shadow is sometimes encountered in patients with ascites and may indicate the presence of fluid in the mediastinum.

Attention was drawn to other abnormal mediastinal radiological appearances, recognition of which is of importance in avoiding diagnostic errors.

\section{CHOLECYSTECTOMY FOR CARDIAC PAIN}

G. E. SOWTON considered the relationship between cardiac symptoms and gall bladder disease both theoretically and clinically, together with results of gall bladder surgery.

A preliminary report on the cardiographic changes of 40 patients undergoing cholecystectomy was produced together with the results of exercise tolerance cardiograms, blood cholesterol levels, and clinical details.

The results of experimental stimulation of the biliary tree during operation, using stronger distension stimuli than have previously been reported, were discussed, with particular reference to the cardiac risk undergone by the patient at operation. 
HISTOLOGICAL ABNORMALITIES IN THE JEJUNUM AND ILEUM IN THE MALABSORPTION SYNDROME

R. HOLMES, D. HOURIHANE, AND C. C. BOOTH (LONDON) described the results of intestinal biopsies in 120 patients using the Crosby biopsy capsule. The specimens were usually examined first under the dissecting microscope and histological sections were then prepared for conventional microscopy.

Thirty patients had idiopathic steatorrhoea. In 18 there was subtotal atrophy of the villi, the biopsies appearing flat and devoid of villi under the dissecting microscope. In the other 12 patients there was partial atrophy, histological sections revealing blunt, squat, and thickened 'villi'; under the dissecting microscope these specimens showed a remarkable ridged appearance which contrasted with the baldness of subtotal atrophy. Biopsies obtained from the ileum might be normal even though there was subtotal atrophy of the jejunal mucosa.
Biopsies from four patients with chronic tropical sprue showed partial villous atrophy and a similar ridged appearance under the dissecting microscope.

The biopsy technique proved invaluable in the differential diagnosis of steatorrhoea. It was also useful in assessing the response to treatment. The procedure was safe and, apart from transient pyrexia after biopsy in two patients, there were no complications.

THE EFFECT OF HAEMATEMESIS ON THE HAEMOPOIETIC SYSTEM

W. M. DAVIDSON (LONDON) said that haematemesis was usually due to primary disease of the oesophagus, stomach, or duodenum; less frequently disease in a neighbouring organ involving the upper part of the alimentary tract; and rarely generalized disease, including diseases of the blood. 